\title{
Impact of laser launch location on the performance of laser tomography and multiconjugate adaptive optics for extremely large telescopes
}

\author{
Luc Gilles, ${ }^{*}$ Lianqi Wang, and Brent L. Ellerbroek \\ Thirty Meter Telescope Observatory Corporation, 1200 East California Boulevard, \\ Mail Code 102-8, Pasadena, California 91125, USA \\ *Corresponding author: Igilles@ caltech.edu \\ Received 31 March 2010; accepted 21 July 2010; \\ posted 9 August 2010 (Doc. ID 126302); published 1 September 2010
}

\begin{abstract}
Laser tomography and multiconjugate adaptive optics are under development for ground-based extremely large telescopes. Continuous wave sodium guide star lasers are planned for these systems, but their use raises some difficulties due to the extended nature of the beacons generated in the mesosphere and their spatiotemporal variability. We describe a performance analysis on the impact of laser launch telescope (LLT) location for the Thirty Meter Telescope (TMT) multiconjugate adaptive optics system. A semianalytical first-order noise propagation calculation is presented, supplemented by end-to-end Monte Carlo physical optics simulations. The principal conclusion of the study is that modestly superior performance is achieved with multiple LLT locations around the primary mirror, compared to a single central LLT behind the secondary mirror, but the largest value of any of these improvements is of the order of $20 \mathrm{~nm} \mathrm{rms}$ for the expected wavefront sensor noise levels, suggesting that the final choice of geometry should depend primarily on the cost and complexity of implementation trade-off. This conclusion is also fully supported by the fact that, for the TMT 70 arcsec laser guide star (LGS) asterism, the fratricide effect reduces the performance of the central launch geometry by only a small amount. The reduction ranges from only a few $\mathrm{nm} \mathrm{rms}$ at zenith to a few tens of $\mathrm{nm}$ at a $45^{\circ}$ zenith angle in the worst case that the effect cannot be calibrated. (C) 2010 Optical Society of America
\end{abstract}

OCIS codes: $\quad 010.1080,010.7350$.

\section{Introduction}

An integral part of next-generation ground-based astronomical telescopes are laser guide star (LGS) adaptive optics (AO) systems. Many systems will utilize multiple mesospheric sodium LGSs in order to implement laser tomography and multiconjugate adaptive optics [1-ㅣㄹ. Continuous wave $(\mathrm{CW})$ sodium guide star lasers are planned for these systems, but their use raises some difficulties. First, the CW beacons are not point sources but rather extended sources along the laser beam propagation directions,

0003-6935/10/31G114-06\$15.00/0

(C) 2010 Optical Society of America due to the thickness of the mesospheric sodium layer. Second, the mean range, density, and shape of the sodium layer fluctuate [4]. Both of these effects call for sophisticated wavefront sensing techniques implementing noise-optimal centroiding algorithms [5] and background processes updating these algorithms as mesospheric and atmospheric turbulence conditions change $[6,7]$.

In this paper, we analyze the impact of laser launch telescope (LLT) location on the performance of the Thirty Meter Telescope (TMT) Narrow Field Infrared Adaptive Optics System (NFIRAOS), which is a dual-conjugate system of order $60 \times 60$ [3]. We take into account the combined effects of wavefront sensor measurement noise arising from 
perspective elongation, atmospheric tomography, and turbulence-induced wavefront sensor nonlinearities. The design of the TMT Laser Guide Star Facility (LGSF) is based on a central launch (CL) concept, in which all guide stars are projected from a single LLT located behind the telescope secondary mirror [3]. In comparison, the European Extremely Large Telescope (E-ELT) design is based on a sidelaunch (SL) architecture, with one LLT per guide star located around the boundary of the telescope primary mirror [8]. Recent simulation results $[9,10]$ suggest that the SL architecture is superior in terms of reducing the wavefront error due to LGS wavefront sensor (WFS) measurement noise, at least for the hexagonal (HEX) LGS asterism adopted for the E-ELT. This result is not obvious, given that the SL configuration increases the maximum extent of guide star elongation by a factor of 2 . On the other hand, this elongation occurs in a different part of the aperture for each guide star.

The goal of this paper is to quantify the magnitude of these effects for the TMT multiconjugate adaptive optics system using a semianalytical geometric noise propagation calculation, supplemented by end-toend Monte Carlo physical optics simulations. We find that modestly superior performance is achieved with multiple LLT locations around the primary mirror, compared to a single central LLT behind the secondary mirror, but the largest value of any of these improvements is of the order of $20 \mathrm{~nm} \mathrm{rms}$ for the expected LGS WFS noise levels, suggesting that the final choice of geometry should depend primarily on the cost and complexity of implementation tradeoff. This conclusion is also fully supported by the fact that, for the TMT 70 arcsec LSG asterism, the fratricide effect reduces the performance of the central launch geometry by only a small amount. The reduction ranges from only a few $\mathrm{nm}$ rms at zenith to a few tens of nm at a $45^{\circ}$ zenith angle in the worst case that the effect cannot be calibrated $[11,12]$. We find also that the performance predicted by an open-loop geometric noise propagation calculation appears to be consistently slightly optimistic, presumably due to the neglect of nonlinearities present in a ShackHartmann WFS with elongated beacons.

The paper has been structured as follows: Section 2 describes the median atmospheric turbulence and mesospheric sodium profiles used to perform the study, as well as the four realizable LLT configurations investigated for TMT. Section 3 discusses the geometrical noise propagation calculation, whereas Section 4 reports on closed-loop Monte Carlo physical optics simulations. Finally, Section $\underline{5}$ concludes the study.

\section{Realizable LLT Configurations Investigated}

The study has been performed for the median Mauna Kea atmospheric turbulence profile described in Table 1 , and for the median mesospheric sodium profile shown in Fig. 1, obtained by averaging 88 contiguous frames recorded every $72 \mathrm{~s}$ by the Purple Crow
Table 1. Median Mauna Kean Atmospheric Turbulence and Wind Profiles ${ }^{a}$

\begin{tabular}{lccllrrr}
\hline Altitude $(\mathrm{km})$ & 0 & 0.5 & 1 & 2 & 4 & 8 & 16 \\
Wind Speed $(\mathrm{m} / \mathrm{s})$ & 5.6 & 5.8 & 6.2 & 7.6 & 13 & 19 & 12 \\
Weights $(\%)$ & 29 & 18 & 6.6 & 7.8 & 14 & 12 & 13 \\
\hline
\end{tabular}

${ }^{a}$ The Fried parameter, the isoplanatic angle, and the Greenwood frequency are, respectively, equal to $r_{0}=19.9 \mathrm{~cm}, \theta_{0}=$ 2.2 arcsec, and $f_{G}=21.7 \mathrm{~Hz}$ at $500 \mathrm{~nm}$ wavelength. The generalized isoplanatic angle for a system with two DMs conjugates to 0 , and $11.2 \mathrm{~km}$ is $\theta_{2}=8.2 \operatorname{arcsec}$ at $500 \mathrm{~nm}$ wavelength.

Lidar system [13]. The FWHM of this profile is about $10 \mathrm{~km}$, which is typical of the values obtained in other investigations [4].

Because of space constraints in the telescope primary mirror support structure, there are only 12 locations around the TMT primary mirror that can accommodate a guide star laser system with an LLT. Four of these locations provide additional space for the support of two lasers. Alternatively, a single LLT can be installed behind the TMT secondary mirror, for use with eight or perhaps more remotely located lasers if the required beam transfer optics system is implemented. These constraints lead to four distinct, realizable LLT configurations that can generate an LGS asterism of either six or eight beacons, respectively, for NFIRAOS and a future multiobject adaptive optics system. These realizable configurations consist of:

- A single LLT located behind the TMT secondary mirror.

- Six LLTs equally spaced around the edge of the TMT primary mirror. Each of these LLTs projects a single laser for the NFIRAOS asterism.

- Four LLTs located at the corners of a rectangle around the edge of the TMT primary mirror. Two of these LLTs would project two lasers each for the NFIRAOS asterism, with the remaining two LLTs projecting a single laser.

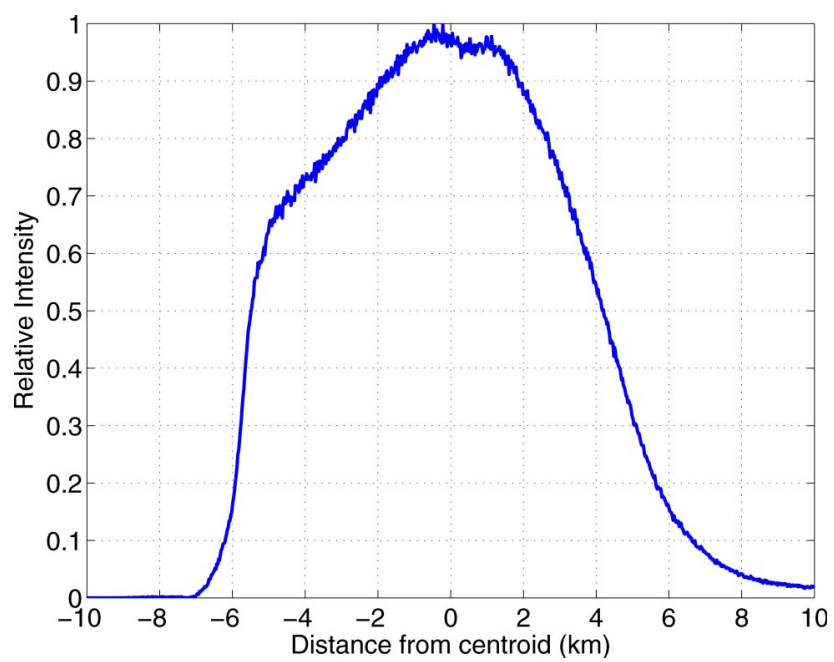

Fig. 1. (Color online) Median mesospheric sodium profile. 

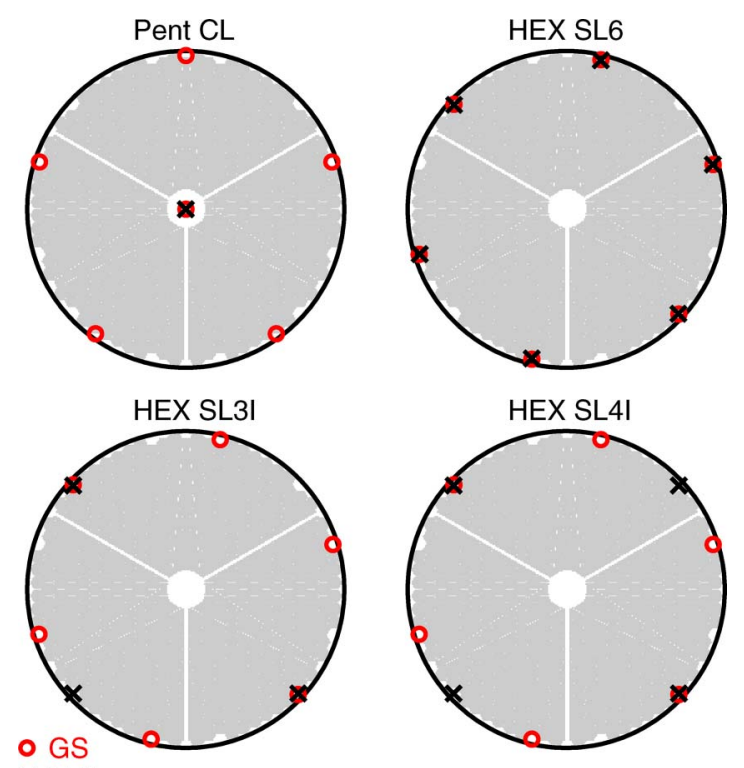

$\times$ LLT

Fig. 2. (Color online) Illustration of the four realizable laser launch telescope (LLT) configurations investigated. Circles indicate the associated guide star (GS) asterism. Each GS is projected by the closest LLT, in all cases.

- Three LLTs located at three of the four corners of the above rectangle. Each of these three LLTs would project two lasers for the NFIRAOS asterism.

These four LLT/asterism configurations are shown in Fig. 2 and are denoted "Pent CL" (NFIRAOS asterism, central launch), "HEX SL3I" (HEX asterism, three irregularly spaced side-launch locations), "HEX SL4I" (HEX asterism, four irregularly spaced sidelaunch locations), and "HEX SL6" (HEX asterism, six side-launch locations). Each guide star (GS) is projected by the closest LLT, in all cases.

As a first step (in order to reduce the size of the trade space), we performed Monte Carlo simulations to determine the preferred size and shape of the LGS asterism for each LLT geometry. These simulations were performed for the nominal LGS WFS signal level of 900 photodetected electrons per subaperture at a sampling rate of $800 \mathrm{~Hz}$ [14]. As shown in Fig. 3, the optimal diameter of the Pent CL geometry was found equal to 70 arcsec, whereas that of the three HEX geometries was found equal to 60 arcsec. Note also that, for the side-launch configurations, the WFE decreases as the number of launch locations increases.

\section{Semianalytical Open-Loop Noise Propagation Analysis}

The tomographic WFE induced by LGS WFS measurement noise is given to first order by $x=E \eta$, where $E$ denotes the minimum variance tomographic estimator and $\eta$ denotes the measurement noise, whose block-diagonal covariance matrix $C_{\eta}$ is assumed to be known and incorporated into $E$. A constrained matched filter computed from a shortexposure Kolmogorov degraded image model has been used to compute $C_{\eta}[\underline{15}, \underline{16}]$, which has the

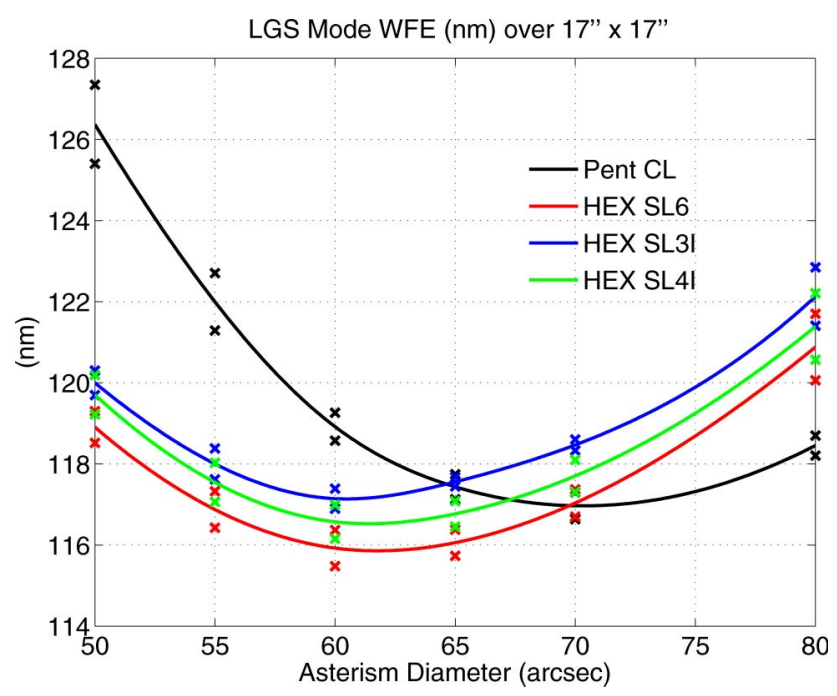

Fig. 3. (Color online) Wavefront error averaged over a $17 \operatorname{arcsec} x$ 17 arcsec field for the different LLT configurations versus asterism diameter. Crosses indicate the amount of scatter as a function of atmospheric turbulence realization. From top to bottom, the curves represent Pent CL, HEX SL3I, HEX SL4I, and HEX SL6.

advantages of minimizing noise propagation and being insensitive to the exact WFS CCD pixel geometry. A readout noise of three electrons per pixel and a signal level of $N_{\text {pde }}=900$ photodetected electrons/ subaperture/1.25 ms has been assumed. This signal level was calculated from the following lidar link equation:

$$
N_{\mathrm{pde}}=\Phi A T_{\mathrm{AOS}} \tau
$$

where $\tau$ denotes the sampling period, $T_{\mathrm{AOS}}$ the equivalent $\mathrm{AO}$ system transmittance (telecope+ WFS optics + detector quantum efficiency), $A$ the subaperture area, and $\Phi$ the photon flux at the ground, itself calculated from the following expression:

$$
\Phi=\left(P_{\mathrm{L}} T_{\mathrm{LGSF}} T_{\mathrm{BQ}}\right)\left(s C_{\mathrm{Na}} \sec (\psi)\right)\left(T_{\mathrm{ATM}}^{\mathrm{sec}(\psi)} / h \sec (\psi)\right)^{2},
$$

where $P_{\mathrm{L}}$ is the CW laser power, $T_{\mathrm{LGSF}}$ the LGSF transmittance, $T_{\mathrm{BQ}}$ the equivalent beam quality transmittance, $s$ the sodium coupling efficiency, $C_{\mathrm{Na}}$ the sodium abundance, $\psi$ the zenith angle, $T_{\mathrm{ATM}}$ the one-way atmospheric transmittance, and $h$ the mean altitude of the sodium layer. The value of $N_{\text {pde }}=900$ is obtained for the following parameters: $\quad \tau=1 / 800 \mathrm{~s}, \quad T_{\mathrm{AOS}}=0.54, \quad A=(0.5)^{2} \mathrm{~m}^{2}$, and $\Phi=5.33 \times 10^{6}$ photons $/ \mathrm{s} / \mathrm{m}^{2}$, itself obtained for $\quad P_{\mathrm{L}}=25 \mathrm{~W}, \quad T_{\mathrm{LGSF}}=0.75, \quad T_{\mathrm{BQ}}=0.8, \quad s=$ 130 photons $/ \mathrm{s} / \mathrm{W} /$ atom $/ \mathrm{m}^{2}, C_{\mathrm{Na}}=3.1 \times 10^{13}$ atoms $/$ $\mathrm{m}^{2}, h=89.4 \times 10^{3} \mathrm{~m}, \psi=0$, and $T_{\mathrm{ATM}}=0.84$. Note that a value of $s=220$ is predicted at Mauna Kea for a circularly polarized laser, pumping both the $\mathrm{D} 2 \mathrm{a}$ and the D2b spectral lines (with $\sim 10 \%$ of the laser power pumping the D2b line) [17]. Such a value of the coupling efficiency yields $\overline{N_{\text {pde }}}=900$ at a 
pessimistic low column density of $C_{\mathrm{Na}}=1.9 \times$ $10^{13}$ atoms $/ \mathrm{m}^{2}$. Note also that measurements at Gadanki (latitude $13.5 \mathrm{~N}$ ) indicate nocturnal variations in the sodium abundance ranging from $C_{\mathrm{Na}}=2 \times 10^{13}$ to $C_{\mathrm{Na}}=7 \times 10^{13}$ [18]. Finally, stellar occultation measurements at latitude $20 \mathrm{~N}$ indicate a median value of $C_{\mathrm{Na}}=3.4 \times 10^{13}$ over one year [19].

Without loss of generality, the open-loop noise analysis has been restricted to the wavefront propagated on-axis, denoted $\psi=H_{x} x$. The piston-removed wavefront variance of $\psi$ for the aperture weighting matrix $W$ is given by the following expressions:

$$
\begin{gathered}
\sigma^{2}=\psi^{\mathrm{T}} W \psi=\operatorname{Tr}\left[\psi \psi^{\mathrm{T}} W\right]=\sum_{i} \sigma_{i}^{2}, \\
\sigma_{i}^{2}=\left[H_{x} E C_{\eta} E^{\mathrm{T}} H_{x}^{\mathrm{T}} W\right]_{i i} .
\end{gathered}
$$

The value of $\sigma_{i}$ has been computed numerically for each point $i$ in the aperture plane for the four LLT configurations using an exact Cholesky factorization/backsubstitution method for the tomographic wavefront reconstruction operator $E$. The resulting maps of wavefront variance are displayed in Fig. 4, clearly evidencing the averaging effects of the sidelaunch configurations. The point-to-point oscillations observed in the maps are due to the sheared aperture-plane reconstruction grid: nodes at subaperture corners propagate less noise than nodes at the middle of subaperture boundaries or at subaperture centers. Results for the rms WFE due to noise are summarized in Table 2. In a closed loop, wavefront variances need to be multiplied by the noise gain $\xi$ of the temporal filter $g(z)$ implemented, where $z=e^{(2 i \pi x)}$. A standard integrator with a two-frame latency, $g(z)=z^{-2} \beta /\left(1-z^{-1}\right)$, has a noise gain equal to

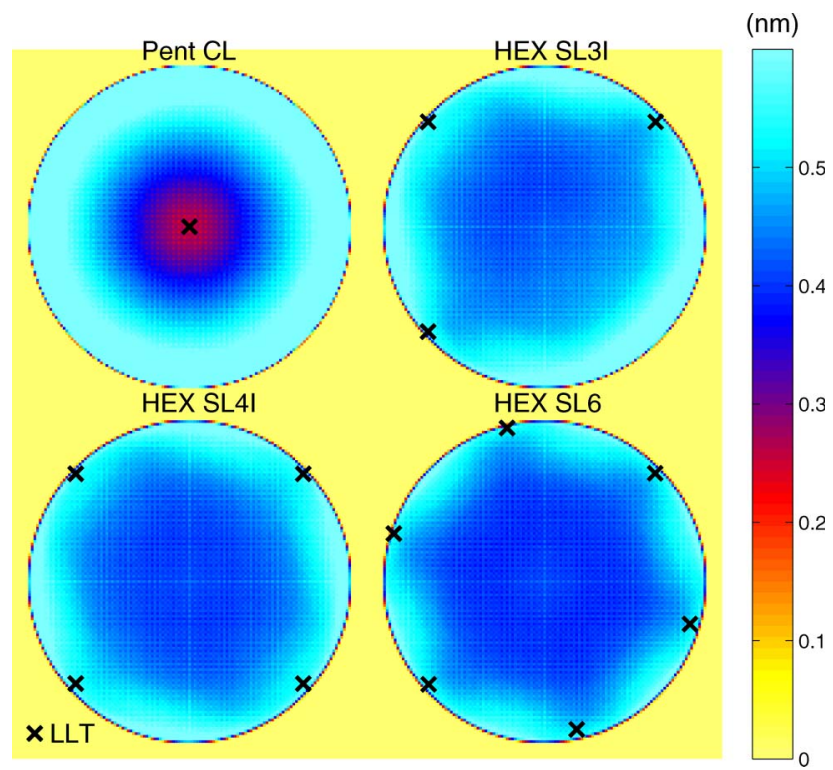

Fig. 4. (Color online) Spatial distribution of the open-loop wavefront variance due to LGS WFS noise.
Table 2. Theoretical rms WFE (nm) due to LGS WFS Noise for the

\begin{tabular}{|c|c|c|c|c|}
\hline & Pent CL & HEX SL3I & HEX SL4I & [ HEX SL6 \\
\hline Open-loop & 61 & -28 & -33 & -37 \\
\hline Closed-loop, $\xi=3 / 5$ & 48 & -22 & -25 & -28 \\
\hline
\end{tabular}
Different LLT Configurations Investigated ${ }^{a}$

${ }^{a}$ The values in each row are quoted as incremental in quadrature with respect to the Pent CL case, and the first column shows the actual rms WFE. Negative values indicate improved performance.

$$
\xi=\int_{-1 / 2}^{1 / 2}\left|\frac{g(z)}{1+g(z)}\right|^{2} \mathrm{~d} x=\frac{-\beta\left(2-\beta^{2}+\beta^{3}\right)}{\left(2-2 \beta+\beta^{2}\right)(\beta+2)(\beta-1)}
$$

which for a typical gain $\beta=1 / 2$ is equal to $\xi=3 / 5$. Note that, as reported in the previous section, for the side-launch configurations, the WFE due to LGS WFS noise decreases as the number of launch locations increases.

\section{Closed-Loop Monte Carlo Simulations}

In this section, verification of the above semianalytical geometric results is performed via end-to-end closed-loop Monte Carlo physical optics simulations of NFIRAOS. Performance was assessed against the LGS WFS signal level. Simulations were conducted during 1000 frames, for signal levels equal to 225, 450,900 , and 1800 photodetected electrons per subaperture per $1.25 \mathrm{~ms}$. The first two cases were run at a sampling frequency of 400 and $600 \mathrm{~Hz}$, respectively, in order to approximately minimize the overall WFE due to the combined effects of measurement noise and servo lag. Results for the LGS mode WFE averaged over a $17 \operatorname{arcsec} \times 17$ arcsec field of view are shown in Fig. 5. More details on the simulation conditions can be found in Ref. [14]. Simulations captured the following combined "fundamental" error

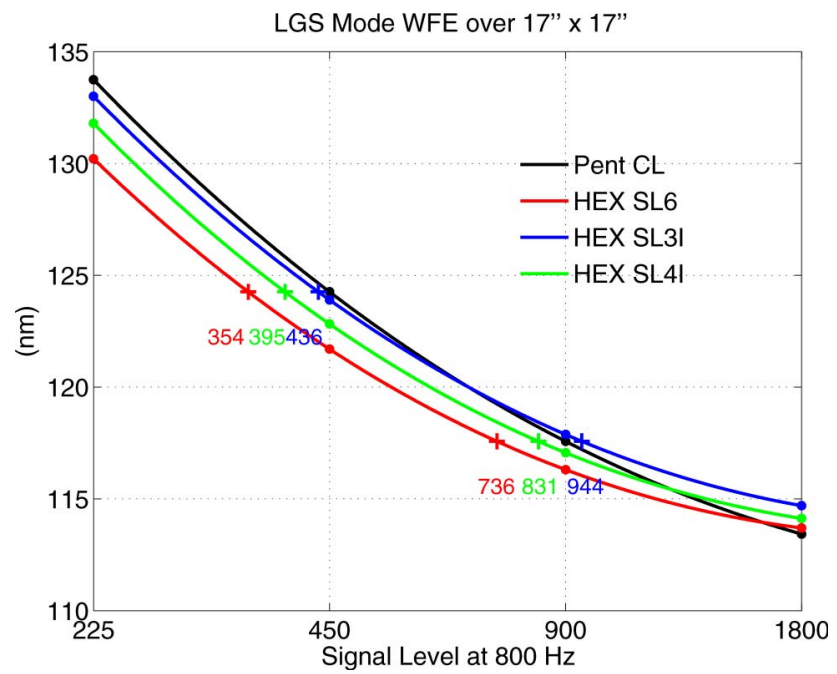

Fig. 5. (Color online) Wavefront error averaged over a $17 \operatorname{arcsec} x$ 17 arcsec field for the different LLT configurations versus LGS WFS signal level quoted as photodetected electrons per subaperture per $1.25 \mathrm{~ms}$. From top to bottom, the curves represent Pent CL, HEX SL3I, HEX SL4I, and HEX SL6. 


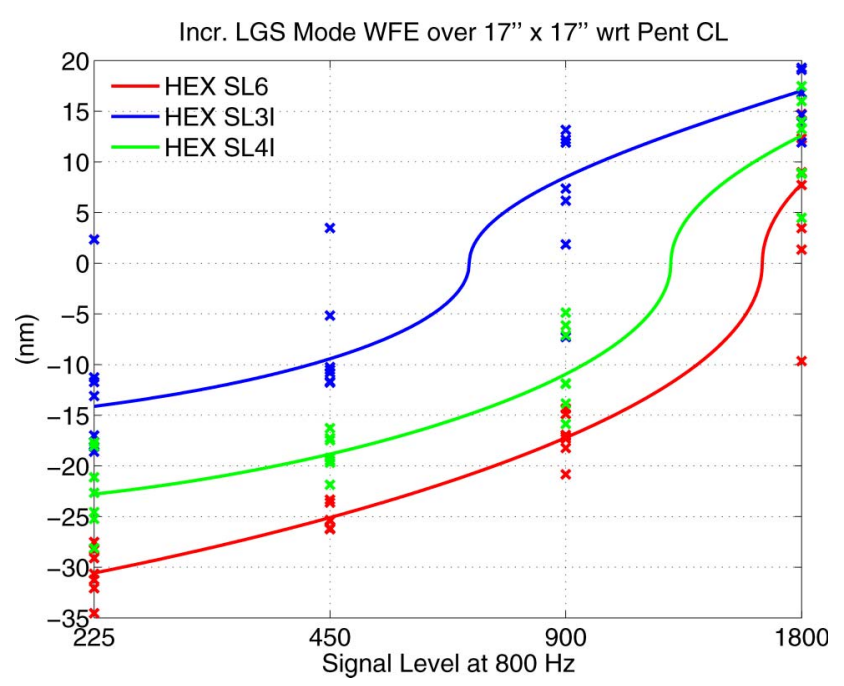

Fig. 6. (Color online) Incremental wavefront error in quadrature with respect the Pent CL case. From top to bottom, the curves represent HEX SL3I, HEX SL4I, and HEX SL6.

terms: (1) DM fitting, (2) DM projection, (3) servo lag, (4) LGS WFS aliasing, (5) tomography, (6) LGS WFS noise, (7) LGS WFS nonlinearity, and (8) the TMT pupil function. Note that the impact of LGS WFS fratricide for the center-launch option is not included, but it has been evaluated in detail in Refs. [11,12]. Figure 6 displays the incremental LGS mode WFE with respect to the Pent CL case. Note that, in distinction with the open-loop noise propagation analysis presented in Section 3, this incremental error captures three combined effects: LGS WFS noise, LGS WFS nonlinearity, and atmospheric tomography. The crosses in the plot quantify the amount of scatter in the estimates as a function of the atmospheric turbulence realization. The main conclusions to be drawn from these simulations are the following:

- At a signal level of 900 photodetected electrons per subaperture per $1.25 \mathrm{~ms}$, HEX SL3I performs $+8 \mathrm{~nm}$ worse than Pent CL, whereas HEX SL4I and HEX SL6 provide modest performance improvements of -12 and $-18 \mathrm{~nm}$, respectively. These values are somewhat smaller than the theoretical predictions made in Table 2, possibly due to the cross coupling of the errors due to noise, nonlinearity, and tomography. Alternatively, equal performance can be achieved with a reduced laser power requirement of $-8 \%$ for HEX SL4I and $-19 \%$ for HEX SL6.

- At a $2 \times$ reduced signal level of 450 photodetected electrons per subaperture per $1.25 \mathrm{~ms}$, all side-launch configurations provide performance superior to Pent CL, with improvements ranging from -10 to $-26 \mathrm{~nm}$. Alternatively, equal performance can be achieved with a reduced laser power requirement of $-4 \%,-13 \%$, and $-22 \%$ for the HEX SL3I, HEX SL4I, and HEX SL6 configurations, respectively.

\section{Conclusions}

We have described a performance analysis on the impact of LLT location for the TMT adaptive optics sys- tem. A semianalytical first-order noise propagation calculation has been presented, supplemented by end-to-end Monte Carlo physical optics simulations. The principal conclusion of the study is that modestly superior performance is achieved with multiple LLT locations around the primary mirror, compared to a single central LLT behind the secondary mirror, but the largest value of any of these improvements is on the order of $20 \mathrm{~nm} r m s$ for the expected LGS WFS noise levels, suggesting that the final choice of geometry should depend primarily on the cost and complexity of implementation trade-off. This conclusion is also fully supported by the fact that, for the TMT 70 arcsec LSG asterism, the fratricide effect reduces the performance of the central launch geometry by only a small amount. The reduction ranges from only a few $\mathrm{nm} \mathrm{rms}$ at zenith to a few tens of $\mathrm{nm}$ at a $45^{\circ}$ zenith angle in the worst case that the effect cannot be calibrated.

The authors gratefully acknowledge the support of the TMT partner institutions: The Association of Canadian Universities for Research in Astronomy (ACURA), the California Institute of Technology, and the University of California. This work was supported, as well, by the Gordon and Betty Moore Foundation, the Canada Foundation for Innovation, the Ontario Ministry of Research and Innovation, the National Research Council of Canada (NRC), the Natural Sciences and Engineering Research Council of Canada, the British Columbia Knowledge Development Fund, the Association of Universities for Research in Astronomy (AURA), and the U.S. National Science Foundation (NSF).

\section{References}

1. E. Diolaiti, J.-M. Conan, I. Foppiani, M. Lombini, C. Petit, C. Robert, L. Schreiber, P. Ciliegi, E. Marchetti, M. Bellazzini, L. Busoni, S. Esposito, T. Fusco, N. Hubin, F. Quiros-Pacheco, A. Baruffolo, S. D’Odorico, J. Farinato, B. Neichel, R. Ragazzoni, C. Arcidiacono, V. Biliotti, G. Bregoli, G. Cosentino, and G. Innocenti, "A preliminary overview of the multiconjugate adaptive optics module for the E-ELT," Proc. SPIE 7015, 70150U (2008).

2. M. Johns, "Progress on the GMT," Proc. SPIE 7012, 70121B (2008).

3. B. Ellerbroek, S. Adkins, D. Andersen, J. Atwood, C. Boyer, P. Byrnes, R. Conan, L. Gilles, G. Herriot, P. Hickson, E. Hileman, D. Joyce, B. Leckie, M. Liang, T. Pfrommer, J.-C. Sinquin, J.-P. Véran, L. Wang, and P. Welle, "Progress toward developing the TMT adaptive optical systems and their components," Proc. SPIE 7015, 70150R (2008).

4. T. Pfrommer, P. Hickson, and C. Y. She, "A large-aperture sodium fluorescence lidar with very high resolution for mesopause dynamics and adaptive optics studies," Geophys. Res. Lett. 36, L15831 (2009).

5. L. Gilles and B. Ellerbroek, "Constrained matched filtering for extended dynamic range and improved noise rejection for Shack-Hartmann wavefront sensing," Opt. Lett. 33, 11591161 (2008).

6. R. Conan, O. Lardière, C. Bradley, G. Herriot, and K. Jackson, "Modeling the Thirty Meter Telescope matched filter based LGS wavefront sensing," Proc. SPIE 7015, 70154S (2008). 
7. O. Lardiére, R. Conan, C. Bradley, G. Herriot, and K. Jackson, "Laser guide star wavefront sensing for TMT: Experimental results of the matched filtering," Proc. SPIE 7015, 70154T (2008).

8. E. Diolaiti, J.-M. Conan, I. Foppiani, M. Lombini, E. Marchetti, C. Petit, C. Robert, L. Schreiber, A. Baruffolo, M. Bellazzini, G. Bregoli, P. Ciliego, G. Cosentino, V. Biliotti, S. D'Odorico, T. Fusco, N. Hubin, S. Meimon, and J.-F. Sauvage, "Towards the phase A review of MAORY, the multi-conjugate adaptive optics module for the E-ELT," in First Adaptive Optics for Extremely Large Telescope Conference (EDP Sciences, 2010), 02007-p1-02007-p5, available online at http://ao4elt.edpsciences.org.

9. M. Tallon, I. Tallon-Bosc, C. Béchet, and E. Thiébaut, "Shack-Hartmann wavefront reconstruction with elongated sodium laser guide stars: Improvements with priors and noise correlations," Proc. SPIE 7015, 70151N (2008).

10. C. Robert, J.-M. Conan, D. Gratadour, C. Petit, and T. Fusco, "Shack-Hartmann tomographic wavefront reconstruction using LGS: analysis of spot elongation and fratricide effect," in First Adaptive Optics for Extremely Large Telescope Conference (EDP Sciences, 2010), 05010-p1-05010-p6, available online at http://ao4elt.edpsciences.org.

11. L. Wang, A. Otarola, and B. Ellerbroek, "Impact of sodium laser guide star fratricide on multi-conjugate adaptive optics systems," J. Opt. Soc. Am. A 27, A19-A28 (2010).

12. L. Wang, A. Otarola, and B. Ellerbroek, "Impact of laser guide star fratricide on TMT MCAO system," Proc. SPIE 7736, 77360G (2010).

13. P. S. Argall, R. J. Sica, O. Vassiliev, and M. M. Mwangi, "Lidar measurements taken with a large-aperture liquid mirror: sodium resonance-fluorescence system," Appl. Opt. 39, 2393-2399 (2000).

14. L. Gilles, L. Wang, and B. Ellerbroek, "Modeling update for the Thirty Meter Telescope laser guide star dual-conjugate adaptive optics system," Proc. SPIE 7736, 77360W (2010).

15. L. Gilles and B. Ellerbroek, "Shack-Hartmann wavefront sensing with elongated sodium laser beacons: Centroiding versus matched filtering," Appl. Opt. 45, 6568-6576 (2006).

16. L. Gilles and B. Ellerbroek, "Constrained matched filtering for extended dynamic range and improved noise rejection for Shack-Hartmann wavefront sensing," Opt. Lett. 33, 1159-1161 (2008).

17. R. Holzlöhner, S. M. Rochester, T. Pfrommer, D. B. Calia, D. Budker, J. M. Higbie, and W. Hackenberg, "Laser guide star return flux simulations based on observed sodium density profiles," Proc. SPIE 7736, 77360V (2010).

18. P. V.Prasanth, V. Sivakumar, S. Sridharan, Y.B. Kumar, H. Bencherif, and D.N. Rao, "Lidar observations of sodium layer over low latitude, Gadinki $\left(13.5^{\circ} \mathrm{N}, 79.2^{\circ} \mathrm{E}\right)$ : Seasonal and nocturnal variations," Ann. Geophys. 27, 3811-3823 (2009).

19. D. Fussen, F. Vanhellemont, C. Bingen, E. Kyrölä, J. Tamminen, V. Sofieva, S. Hassinen, A. Seppälä, P. Verronen, J.-L. Bertaux, A. Hauchecorne, F. Dalaudier, J.-B. Renard, R. Fraisse, O. F.d'Andon, G. Barrot, A. Mangin, B. Théodore, M. Guirlet, R. Koopman, P. Snoeij, and L. Saavedra, "Global measurement of the mesospheric sodium layer by the star occultation instrument GOMOS," Geophys. Res. Lett. 31, L24110 (2004). 